\section{Mutually exclusive mutations of the Pten and ras pathways in skin tumor progression}

\author{
Jian-Hua Mao, ${ }^{1}$ Minh D. To, ${ }^{1}$ Jesus Perez-Losada, ${ }^{1}$ \\ Di Wu, Reyno Del Rosario, and Allan Balmain ${ }^{2}$ \\ University of California at San Francisco Comprehensive \\ Cancer Center, San Francisco, California 94143, USA
}

Pten heterozygous $\left(\right.$ Pten $\left.^{+/}\right)$mice develop increased papilloma numbers and show decreased carcinoma latency time in comparison with controls after skin treatment with dimethyl benzanthracene (DMBA) and tetradecanoyl-phorbol acetate (TPA). $\mathrm{H}$-ras mutation is normally a hallmark of DMBA-TPA-induced skin tumors, but 70\% of carcinomas from Pten $^{+/-}$mice do not exhibit this mutation, and in all cases have lost the wild-type Pten allele. Tumors that retain the Pten wild-type allele also have $\boldsymbol{H}$-ras mutations, indicating that activation of $\boldsymbol{H}$ ras and complete loss of Pten are mutually exclusive events in skin carcinomas. Mitogen-activated protein kinase (MAPK) is consistently activated in the tumors with $\mathrm{H}$-ras mutations, but is strongly down-regulated in Pten $^{-/-}$tumors, suggesting that this pathway is dispensable for skin carcinoma formation. These data have important implications in designing individual therapeutic strategies for the treatment of cancer.

Received April 21, 2004; revised version accepted June 2, 2004.

The Pten tumor suppressor gene encodes a dual protein and lipid phosphatase that antagonizes PI3K/Akt signaling, a pathway that triggers a cascade of responses important for tumor development (Vivanco and Sawyers 2002). Somatic alterations that negate Pten function are commonly detected in a number of human tumor types (Li et al. 1997; Steck et al. 1997). In addition, germ-line mutations in Pten have been described in heritable cancer risk syndromes, Cowden disease (CD) and BannayanZonana syndrome (Liaw et al. 1997; Marsh et al. 1997). Homozygous deletion of Pten results in embryonic lethality (Di Cristofano et al. 1998; Stambolic et al. 1998; Podsypanina et al. 1999), indicating that it is a developmentally essential gene. Mice that are heterozygous for Pten are viable, but they show abnormal pathological features and are susceptible to developing tumors in a number of tissues (Di Cristofano et al. 1998; Podsypanina et al. 1999). The skin of these mice shows focally increased epidermal thickness due to hyperkeratosis (Di Cristofano et al. 1998), a feature commonly observed with CD patients. The association of squamous

[Keywords: Pten; ras; Akt; skin; papilloma; carcinoma]

${ }^{1}$ These authors contributed equally to this work.

${ }^{2}$ Corresponding author.

E-MAIL abalmain@cc.ucsf.edu; FAX (415) 502-6779.

Article and publication are at http://www.genesdev.org/cgi/doi/10.1101/ gad.1213804. cell carcinomas with CD has also been reported /Camisa et al. 1984; Hildenbrand et al. 2001), but these lesions were not observed in Pten ${ }^{+/-}$animals. On the other hand, $100 \%$ of mice with complete Pten deficiency, and a proportion with Pten heterozygosity, in their keratinocytes spontaneously develop papillomas and squamous cell carcinomas (Suzuki et al. 2003), indicating that Pten is an important suppressor of skin tumorigenesis.

We have used dimethyl benzanthracene (DMBA)-tetradecanoyl-phorbol acetate (TPA)-induced skin carcinogenesis to study the molecular mechanisms that underlie the development of this malignancy. A signature of DMBA treatment is an activating mutation in $H$-ras, which is the most common initiating event for tumor formation (Balmain et al. 1984; Quintanilla et al. 1986). We demonstrate here that most skin carcinomas from Pten $^{+/-}$mice do not have this signature mutation, but instead show complete loss of Pten by deletion of the wild-type allele. These genetic changes are correlated with major differences in signaling through the mitogenactivated protein kinase (MAPK) and PI3K/Akt pathways.

\section{Results and Discussion}

To investigate the role of Pten in skin carcinogenesis, we treated an equal number of Pten $^{+/-}$mice and wild-type littermates with DMBA and TPA. We used germ-line Pten $^{+/-}$mice (Di Cristofano et al. 1998), as opposed to tissue-specific knockout (KO) models (Suzuki et al. 2003) to better recapitulate the condition of CD. The basal level of Pten protein is clearly reduced in the skin of these heterozygous animals (Fig. 1A), and this is relevant given that Pten expression is rapidly up-regulated, both at the RNA and protein levels, in response to TPA treatment (Fig. 1B). The increase in levels of a tumor suppressor protein such as Pten is intriguing in view of the fact that TPA treatment strongly induces proliferation in the epidermis. A similar situation is, however, seen with other negative growth regulators such as TGFß (Akhurst et al. 1988), which are also induced in response to TPA treatment, possibly as part of a mechanism that ensures a regulated return to homeostatic equilibrium within the epidermis. In light of these observations, we predicted Pten $^{+/-}$mice to be more susceptible to DMBA-TPA-induced skin carcinogenesis, although the risk of spontaneously developing skin cancer was not noticeably altered (Di Cristofano et al. 1998; Podsypanina et al. 1999). Both Pten $^{+-}$and wild-type mice developed papillomas after 7-8 wk of DMBA-TPA treatment. However, the papilloma number was significantly higher in $\mathrm{Pten}^{+/-}$ animals in comparison with wild type in both males and females (Fig. 1C). By $20 \mathrm{wk}$ posttreatment, the mean number of papillomas per mouse was more than threefold higher in Pten $^{+/}$mice $(p<0.0001)$, indicating that loss of one Pten allele renders animals more susceptible to DMBA-TPA-induced skin carcinogenesis.

Papillomas are generally benign, but a small number eventually progresses to the carcinoma stage. Mice were monitored for carcinoma development for up to $60 \mathrm{wk}$ post-DMBA treatment. We observed carcinomas on Pten $^{+/-}$mice as early as 20 wk post-DMBA treatment (Fig. 1D). By 44 wk posttreatment, carcinomas were found on all Pten $^{+/-}$animals, whereas $>70 \%$ of the wildtype littermates remained carcinoma free. The decrease 
A

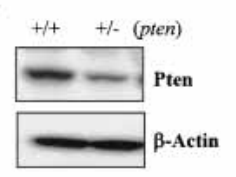

B

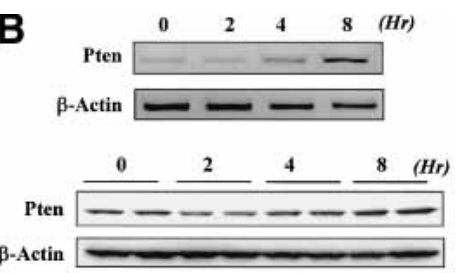

C

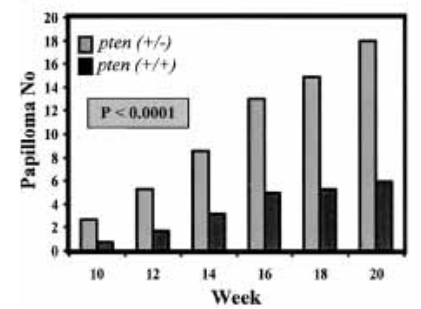

D

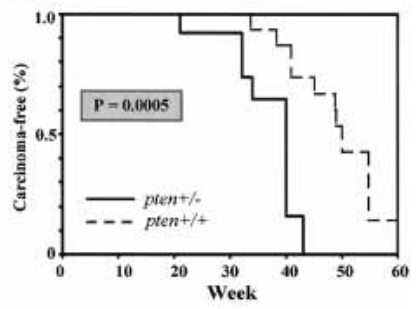

Figure 1. Pten heterozygosity increases skin cancer susceptibility. (A) Pten protein expression in the skin of wild-type and Pten $^{+/}$ mice. (B) Expression of Pten is induced in wild-type mice at RNA $($ top $)$ and protein (bottom) levels at indicated time points after TPA treatment. Pten protein levels in skin of two different mice are shown at each time point. $(C)$ Average papilloma number per mouse at the indicated time points after DMBA-TPA treatment. $(D) \mathrm{La}-$ tency of carcinoma onset.

in carcinoma latency period in Pten $^{+/-}$versus wild-type mice was highly significant $(p<0.005)$, indicating that an impartial loss of Pten function not only increased susceptibility to tumor initiation, but also accelerated tumor progression.

A two-hit mechanism to inactivate tumor suppressor genes has been well documented, and Pten appears to follow this paradigm in many tumor types. We examined the status of Pten in skin tumors using microsatellite markers at, and also proximal and distal to, the Pten genomic locus. In 15 papillomas and 12 carcinomas from wild-type mice, we did not detect any deletions at the Pten locus. In contrast, loss of heterozygosity (LOH) was observed in papillomas and a high number of carcinomas from Pten $^{+/-}$mice (Fig. 2A). In many instances, LOH extends to markers as far as $20 \mathrm{cM}$ from the Pten locus, and may reflect a loss of the entire chromosome 19 . However, LOH at adjacent markers did not occur independently of LOH at the Pten locus, indicating that Pten is the primary target for $\mathrm{LOH}$ in these tumors.

Loss of expression due to regulatory mutations or epigenetic events is one mechanism of inactivating gene activity. However, RT-PCR analysis indicated that the Pten transcript is present in all papillomas and carcinomas from wild-type animals (Fig. 2B). Pten protein is also clearly detectable in these papillomas and carcinomas (Fig. 3A). We sequenced the entire coding region of Pten in these tumors and did not detect any mutation. In papillomas and carcinomas from Pten $^{+/-}$animals, the presence or absence of the Pten wild-type transcript correlated with Pten protein status, and these were in agreement with the microsatellite results. In the majority of carcinoma samples, the wild-type Pten transcript is clearly absent (Fig. 2B). On the other hand, the transcript corresponding to the $\mathrm{KO}$ allele is retained in all papillomas and carcinomas, indicating that LOH selectively targets the chromosome that carries the wild-type Pten allele.
The incidence of Pten LOH in papillomas was 25\% (5/20), which is significantly lower than the $66 \%(6 / 9)$ we observed for carcinomas $(p<0.05)$, indicating that a complete loss of Pten efficiently drives the conversion of benign papillomas to malignant carcinomas. The concept of subtypes of papillomas with high or low malignant potential has been previously described (Hennings et al. 1985), with those having the higher potential for malignant conversion originating from hair follicle stem cells (Perez-Losada and Balmain 2003). One interpretation of our data is that Pten may be important in regulating epidermal stem cell proliferation, similar to that described for neural stem cells (Groszer et al. 2001). In this respect, a complete loss of Pten could result in an increase in the number of hair follicle stem cells and thus the number of potential targets for malignant skin tumor development. In support of this idea, animals with keratinocyte-specific Pten deficiency do indeed have increased hair follicles and decreased interfollicular epidermis (Suzuki et al. 2003). Alternatively, complete loss of Pten may be able to drive progression regardless of the cell of origin.

A lack of Pten alterations in tumors from wild-type mice was a surprising finding because homozygous inactivation of Pten in keratinocytes can clearly cause skin cancer (Suzuki et al. 2003). On the other hand, our data
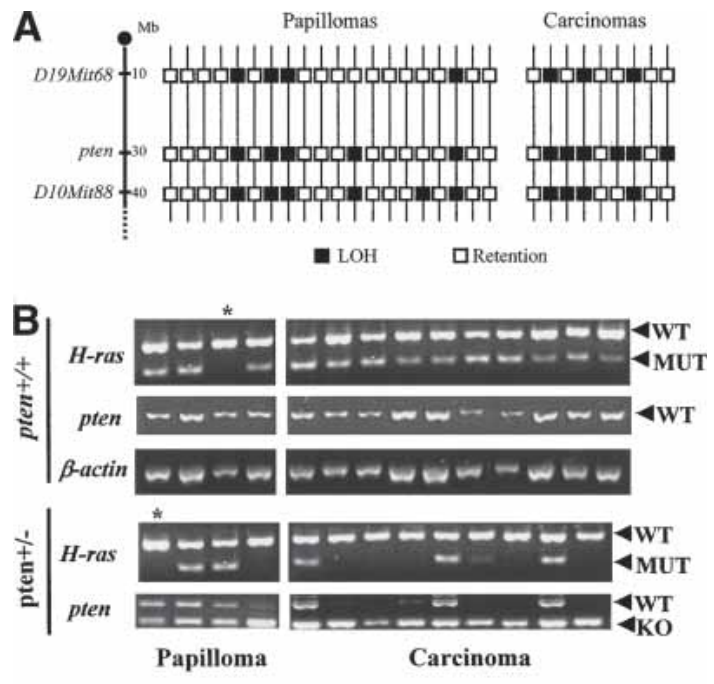

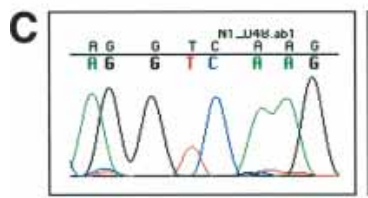

WT

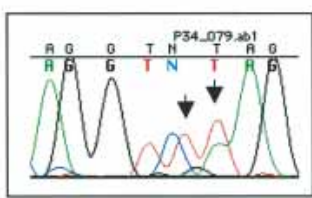

CAA > TTA
Figure 2. H-ras and Pten alterations are mutually exclusive. $(A)$ LOH analysis of tumors from Pten ${ }^{+/-}$mice using the indicated microsatellite markers on chromosome 19 , as obtained from the Celera database. Papilloma \#16 (fifth from the right) shows loss of distal markers on chromosome 19, but is wild type at Pten, and has a mutation in $H$-ras (Table 1). (B) Papillomas and carcinomas from wild type (top panel) and Pten ${ }^{+/-}$(bottom panel) were analyzed for $H$-ras mutation by digestion of PCR products as described in text, and for Pten expression by RT-PCR. Asterisk indicates samples with TTA alteration, as opposed to the more common CTA, at codon 61 of $\mathrm{H}$-ras, and hence was not digested by XbaI. (C) Chromatogram showing the CAA $\rightarrow$ TTA alteration at codon 61 of $H$-ras that occurred in a small number of papillomas. 

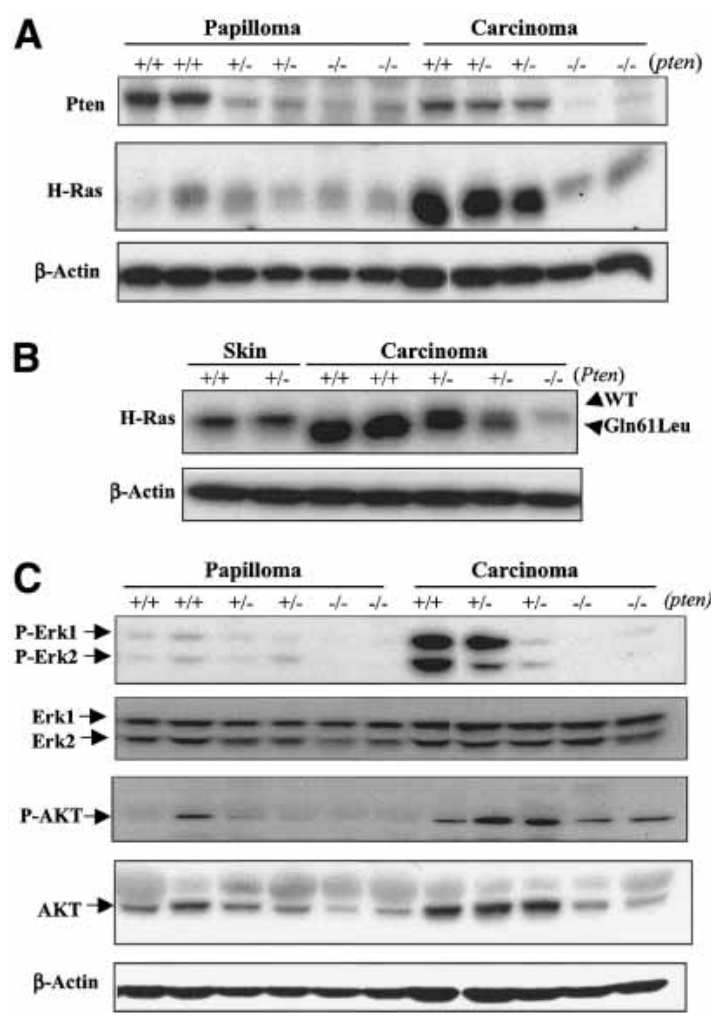

Figure 3. H-ras activation and Pten inactivation elicit differential biochemical responses. The status of pten for each tumor, as determined by microsatellite and RT-PCR analysis, is indicated. $(A)$ Pten and $\mathrm{H}$-Ras protein levels in papillomas and carcinomas. $(B)$ Separation of wild-type and mutant (Gln61Leu) H-Ras protein in carcinomas by high-percentage SDS-PAGE. (C) Analysis of Erk and Akt activation in papillomas and carcinomas using antibodies to detect phosphorylated and total protein, as indicated.

are consistent with findings in human skin tumors, where a lack of somatic Pten alterations has also been reported (Kubo et al. 1999). It is possible that inactivation of both Pten alleles is required during skin carcinogenesis, a situation that is more favorable when the first event has already occurred through germ-line transmission, as is the case with $\mathrm{CD}$ and $\mathrm{Pten}^{+/-}$mice. In this respect, genetic alterations at other loci may substitute for Pten inactivation in tumors from wild-type mice and may even preclude the need for Pten LOH in some tumors from Pten $^{+/-}$animals.

Activating mutations in $H$-ras, usually a CAA-to-CTA change at codon 61, occur at a high frequency in DMBAinitiated skin tumors (Balmain et al. 1984; Quintanilla et al. 1986). H-Ras has a stimulatory effect on PI3K/Akt signaling; thus its activation could occur in place of Pten inactivation. We analyzed skin tumor DNA for H-rasactivating mutations, taking advantage of the XbaI restriction site created by the CTA mutation (Nagase et al. 2003). Activating $H$-ras mutations were detected in all 10 carcinomas from wild-type animals (Fig. 2B). In two papillomas where the mutant product was not detected, DNA sequencing revealed a TTA alteration at codon 61 in place of the more common CTA (Fig. 2C). Interestingly, both CTA and TTA alterations resulted in the same Gln61Leu amino acid change, pointing to impor- tance and selectivity of this residue in activated H-Ras protein. Taken together, our data showed that the codon 61-activating $H$-ras mutation occurs in every DMBATPA-induced papilloma and carcinoma from wild-type mice.

In contrast, the majority of skin carcinomas derived from $\mathrm{Pten}^{+/-}$mice did not carry this $\mathrm{H}$-ras mutation, and all of the tumors in this category had undergone Pten LOH. We did not detect any mutations in $H$-ras despite sequencing the entire coding region. Because mutations in K-ras have been found in skin tumors from DMBATPA treated $H$-ras KO mice (Ise et al. 2000), we also sequenced the entire coding region of $K$-ras in these carcinomas and did not detect any mutation. There is therefore a highly significant correlation between the absence of ras mutations in carcinomas and the loss of the wildtype Pten allele. Table 1 summarizes results of Pten and $H$-ras status in papillomas and carcinomas from wildtype and $\mathrm{Pten}^{+/-}$mice. It can be seen that although the majority of the benign papillomas in $\mathrm{Pten}^{+-}$mice still had activated $H$-ras in the usual manner, $~ 30 \%$ had lost the Pten allele. Of these, two had both activated H-ras and loss of Pten, in contrast to the situation seen in the carcinomas. This may indicate that papillomas carrying both mutations are less likely to progress to malignancy than those showing complete loss of Pten.

Table 1. Summary of H-ras and pten alterations

\begin{tabular}{|c|c|c|c|c|c|}
\hline Pten(+/-) & H-ras & Pten & Pten $(+/+)$ & $H$-ras & Pten \\
\hline Papilloma & & & Papilloma & & \\
\hline 1 & MUT $^{\mathrm{a}}$ & WT & 1 & MUT & WT \\
\hline 2 & MUT & WT & 2 & MUT $^{\mathrm{a}}$ & WT \\
\hline 3 & MUT & WT & 3 & MUT & WT \\
\hline 4 & MUT & WT & 4 & MUT & WT \\
\hline 5 & MUT & LOSS & 5 & MUT & WT \\
\hline 6 & MUT & WT & 6 & MUT & WT \\
\hline 7 & WT & LOSS & 7 & MUT & WT \\
\hline 8 & MUT & LOSS & 8 & MUT & WT \\
\hline 9 & MUT & WT & 9 & MUT & WT \\
\hline 10 & MUT & WT & 10 & MUT & WT \\
\hline 11 & MUT & WT & 11 & MUT & WT \\
\hline 12 & WT & LOSS & 12 & MUT & WT \\
\hline 13 & MUT & WT & 13 & MUT & WT \\
\hline 14 & MUT & WT & 14 & MUT $^{\mathrm{a}}$ & WT \\
\hline 15 & MUT & WT & 15 & MUT & WT \\
\hline 16 & MUT & WT & & & \\
\hline 17 & MUT & WT & Carcinoma & & \\
\hline 18 & WT & LOSS & 1 & MUT & WT \\
\hline 19 & MUT & $\mathrm{WT}$ & 2 & MUT & WT \\
\hline \multirow[t]{2}{*}{20} & MUT & WT & 3 & MUT & WT \\
\hline & & & 4 & MUT & WT \\
\hline Carcinoma & & & 5 & MUT & WT \\
\hline 1 & MUT & WT & 6 & MUT & WT \\
\hline 2 & WT & LOSS & 7 & MUT & WT \\
\hline 3 & WT & LOSS & 8 & MUT & WT \\
\hline 4 & WT & LOSS & 9 & MUT & WT \\
\hline 5 & MUT & WT & 10 & MUT & WT \\
\hline 6 & WT & LOSS & & & \\
\hline 7 & WT & LOSS & & & \\
\hline 8 & MUT & WT & & & \\
\hline 9 & WT & LOSS & & & \\
\hline
\end{tabular}

${ }^{a}$ Codon 61 mutation CAA $\rightarrow$ TTA.

(WT) Wild type; (MUT) mutant. 
The strong up-regulation in H-ras protein level in carcinoma demonstrates the importance of this gene in papilloma-to-carcinoma conversion (Fig. 3A). The mechanism underlying this increase in expression appears to be at the level of gene copy number (Nagase et al. 2003). By using high-percentage polyacrylamide SDS gels to discriminate between mutant and wild-type H-Ras protein by mobility, we could show that it is the oncogenic form of $\mathrm{H}$-Ras protein that is selectively up-regulated in carcinomas (Fig. 3B). In addition, the extent of up-regulation is stronger in tumors with intact Pten than in those that are Pten heterozygous. In carcinomas with complete Pten inactivation, not only is there a lack of $H$-ras-activating mutation, but there is also no up-regulation at the expression level. On the basis of these results, it appears that Pten inactivation confers similar advantages to activated $H$-ras and its subsequent overexpression, at least at the stage of carcinomas. This could account for the decreased carcinoma latency time in $\mathrm{Pten}^{+/-}$mice because $\mathrm{LOH}$ involving the wild-type Pten allele would be the single limiting event, whereas with $H$-ras there is a requirement for mutagenesis as well as allele-specific duplication or amplification (Nagase et al. 2003).

We analyzed the status of downstream targets of $\mathrm{H}$ Ras and Pten in order to better understand the biochemical consequences of their alterations. Several lines of evidence suggest that the activation of Erk-1 and Erk-2 MAPK proteins, which are important components of the H-Ras network, can also be dependent on the status of Pten (Shan et al. 2000; Suzuki et al. 2001, 2003). Despite relatively similar levels of total Erk proteins, the phosphorylated or active forms of both Erk proteins (P-Erk) were only detectable in papillomas or carcinomas that do not have complete Pten inactivation (Fig. 3C) and are most likely a direct consequence of the oncogenic H-Ras protein present in these tumors (see earlier). Even in these cases, the level of P-Erk proteins was barely detectable in papillomas. The Erk proteins are more strongly activated in carcinomas, which are consistent with the up-regulated mutant H-Ras expression in these malignant tumors, but the extent of activation can be variable. The almost complete lack of P-Erk in papillomas and carcinomas with complete Pten inactivation may be a signature of these tumors, and suggests that Erk activation is dispensable in skin carcinogenesis or at least during papilloma-to-carcinoma conversion.

Central to the tumor-suppressing function of Pten is the inhibition of Akt signaling (Stambolic et al. 1998). In contrast, H-Ras activates PI3K activity, which in turn stimulates the Akt pathway (Cox and Der 2003). We observed an increasing trend in levels of phosphorylated Akt (P-Akt) from papillomas to carcinomas as previously reported (Fig. 3C; Segrelles et al. 2002), suggesting that Akt activation is important for carcinoma development and consistent with the role of Akt signaling in promoting cell proliferation and survival (Vivanco and Sawyers 2002). Interestingly, whereas many studies with cultured cells have shown regulation of Akt signaling in response to Pten alterations to be largely at the level of phosphorylation (Stambolic et al. 1998; Suzuki et al. 2003), we found that in papilloma and particularly carcinoma specimens the regulation appears to occur at the level of total Akt protein as well. Tumors with retention of the wild-type Pten allele and carrying $H$-ras mutations in general had higher levels of total Akt protein. It is possible that these higher levels may be a consequence of the activity of Ras/MAP in inducing cell death (Deng et al. 2004), causing a feedback response leading to up-regulation of Akt-mediated survival signals. A dynamic balance between these opposing pathways may explain why no tumors carrying both genetic alterations have progressed to malignancy, whereas those with only Pten loss, and no MAPK activity, have high malignant potential. These results may be relevant to prognosis of human cancer carrying both kinds of genetic changes, which may be expected to have a more indolent growth pattern.

A lack of concurrent Pten alteration and N-ras or K-ras mutation has been reported for melanoma cell lines (Tsao et al. 2000) and endometrial cancer (Ikeda et al. 2000), respectively. However, the relationship between these mutational events is still unclear, and some melanomas or uncultured cells have been reported to have both mutations in B-raf and loss of Pten (Tsao et al. 2004). In this study, we have excluded the possibility that the common sites of activation of $K$-ras or $B$-raf (Davies et al. 2002) are mutated in the tumors that show complete loss of Pten (data not shown). We conclude that Pten inactivation and $H$-ras activation are mutually exclusive in malignant skin carcinomas, suggesting that these events are genetically redundant, and that alteration in both genes does not confer a further advantage. It is also possible that $H$-ras activation and Pten loss is in fact disadvantageous when these events occur together in the same cell. We detected two papillomas with both $H$-ras mutation and Pten inactivation, but no carcinomas with both changes. Although these numbers are small, papillomas with both genetic alterations are possibly at a relative disadvantage for tumor progression to carcinomas, possibly due to reported effects of activated ras in induction of apoptosis (Chang et al. 2003) or growth arrest (Serrano et al. 1997).

These observations of discrete pathways leading to malignancy in this model system have important implications for the design of therapeutic strategies for the treatment of skin cancer as well as other cancers with frequent Pten and/or ras alterations. Tumors with complete loss of Pten and low levels of signaling through the Ras/Raf/Erk pathway will obviously not respond to small molecule drugs that inhibit these targets. Such treatments may even be detrimental, because they may alter the balance between cell death and survival pathways in cancers carrying both mutations.

\section{Materials and methods}

Mice and chemical carcinogenesis

Pten $^{+/-}$mice (C57BL/6), provided by Dr. P.P. Pandolfi (Memorial SloanKettering Cancer Center, New York; Di Cristofano et al. 1998), were crossed with FVB/N to generate F1 wild-type and Pten $^{+/-}$mice. To induce skin carcinogenesis, we used a single dose of DMBA treatment followed by twice-weekly application of TPA for $20 \mathrm{wk}$ as described (Balmain et al. 1984; Quintanilla et al. 1986). Papilloma number was recorded up to 20 wk and carcinoma development monitored up to $60 \mathrm{wk}$ post-DMBA treatment. Mice were killed if moribund, if the tumor load was excessive, if any individual tumor exceeded $1.5 \mathrm{~cm}$ in diameter, or at the termination of the experiment. Surgically removed tumors were immediately snap-frozen in liquid nitrogen, and stored at $-70^{\circ} \mathrm{C}$. We scored tumors as carcinomas or papillomas on the basis of their morphological appearance and histological features.

$D N A / R N A$ and protein preparation

Tumors were ground into a fine powder in liquid nitrogen and aliquotted for DNA and RNA extraction and protein lysate preparation. DNA was isolated using standard phenol/chloroform extraction following an over- 
night $55^{\circ} \mathrm{C}$ incubation with Proteinase K. Total RNA was purified using TRIzol Reagent (Invitrogen). For total protein lysates, powdered tumors were incubated in ice-cold lysis buffer $(50 \mathrm{mM}$ Tris-Cl at $\mathrm{pH} 7.4,2 \mathrm{mM}$ EDTA, $150 \mathrm{mM} \mathrm{NaCl}, 1 \%$ NP-40, 0.5\% Triton X-100, 0.1\% SDS) supplemented with Complete Mini Protease Inhibitor Cocktail (Roche). Soluble protein lysates were quantified using BCA Protein Assay kit (Pierce).

\section{LOH analysis}

Tumor and corresponding normal DNA was used for LOH studies with microsatellite markers at the Pten locus and flanking regions chosen from the Celera database. PCR amplification was performed in $20-\mu \mathrm{L}$ volume containing $1 \times$ PCR buffer (Bioline), $200 \mu \mathrm{M}$ of each dNTP (Pharmacia), $6.6 \mu \mathrm{M}$ of each primer (Qiagen Operon), $1.5 \mathrm{mM} \mathrm{MgCl}_{2}$, 2 units of Taq polymerase (Bioline), and $80 \mathrm{ng}$ of DNA. Amplifications were initially denatured for $3 \mathrm{~min}$ at $94^{\circ} \mathrm{C}$, followed by 35 cycles of $30 \mathrm{sec}$ at $94^{\circ} \mathrm{C}, 30 \mathrm{sec}$ at $55^{\circ} \mathrm{C}$ or $52^{\circ} \mathrm{C}$, and $30 \mathrm{sec}$ at $72^{\circ} \mathrm{C}$. PCR products were electrophoresed in $4 \%(3 \%$ NuSieve $/ 1 \%$ normal) agarose gel and visualized by ethidium bromide staining.

\section{Pten expression analysis}

RT-PCR was performed using Thermoscript RT-PCR (GIBCO-BRL). Mutation analysis of the Pten coding sequence was performed by direct sequencing of gel-purified PCR products of tumor cDNA. Primer pairs used in the analysis were Pten $1 F$ ( $5^{\prime}$-CATCTCTCTCCTCCTTTTTCT TCA-3') and Pten1R (5'-CAAACATCATCTTGTGAAACAGC-3'), Pten2F (5'-GGCAAATTTTTAAAGGCACAAG-3') and Pten2R (5'-AAGTTGA ACTGCTAGCCTCTGG- $\left.3^{\prime}\right)$, and Pten3F (5'-ACTTTGAGTTCCCTCA GCCA-3') and Pten3R (5'-TTCATGGTATTTTATCCCTCTTGA-3').

\section{Immunoblotting}

Equal amounts of proteins were resolved by SDS-PAGE and transferred to polyvinylidene fluoride (PVDF) membrane using standard protocol. We used $18 \%$ polyacrylamide gel in order to distinguish between wild-type and codon $61 \mathrm{H}$-Ras mutant protein. Rabbit polyclonal antibody to phospho-Ser 473-PKB/AKT was a gift from Dr. David Stokoe (University of California at San Francisco). We purchased Pten (A2B1), H-Ras (C-20), and ERK1 (C-16) antibodies from Santa Cruz Biotechnology, and P-Erk (\#9101) and Akt (\#9272) antibodies from Cell Signaling Technology.

\section{Statistical analysis}

SPSS statistical package was used for all statistical analysis. We used the nonparametric test to compare papilloma number and the Kaplan-Meire method to compare latency in progression to carcinoma.

\section{Acknowledgments}

These studies were supported by NCI grant U01 CA84244 to A.B. The UCSF Cancer Center Genome Core was essential for the sequencing. Special thanks go to the University of California at San Francisco animal house staff, and to Dr. P.P. Pandolfi for kindly providing us with Pten KO mice. J.-H.M. is a recipient of a Leukemia \& Lymphoma Society Fellowship. J.P.-L. has a Fellowship from the Ministerio de Educacion y Ciencia of Spain.

The publication costs of this article were defrayed in part by payment of page charges. This article must therefore be hereby marked "advertisement" in accordance with 18 USC section 1734 solely to indicate this fact.

\section{References}

Akhurst, R.J., Fee, F., and Balmain, A. 1988. Localized production of TGF- $\beta$ mRNA in tumour promoter-stimulated mouse epidermis. $\mathrm{Na}$ ture 331: 363-365.

Balmain, A., Ramsden, M., Bowden, G.T., and Smith, J. 1984. Activation of the mouse cellular Harvey-ras gene in chemically induced benign skin papillomas. Nature 307: 658-660.

Camisa, C., Bikowski, J.B., and McDonald, S.G. 1984. Cowden's disease. Association with squamous cell carcinoma of the tongue and perianal basal cell carcinoma. Arch. Dermatol. 120: 677-678.

Chang, F., Steelman, L.S., Shelton, J.G., Lee, J.T., Navolanic, P.M., Blalock, W.L., Franklin, R., and McCubrey, J.A. 2003. Regulation of cell cycle progression and apoptosis by the Ras/Raf/MEK/ERK pathway Int. J. Oncol. 22: 469-480.

Cox, A.D. and Der, C.J. 2003. The dark side of Ras: Regulation of apoptosis. Oncogene 22: 8999-9006.

Davies, H., Bignell, G.R., Cox, C., Stephens, P., Edkins, S., Clegg, S., Teague, J., Woffendin, H., Garnett, M.J., Bottomley, W., et al. 2002. Mutations of the BRAF gene in human cancer. Nature 417: 949-954.

Deng, Q., Liao, R., Wu, B.L., and Sun, P. 2004. High intensity ras signaling induces premature senescence by activating p38 pathway in primary human fibroblasts. J. Biol. Chem. 279: 1050-1059.

Di Cristofano, A., Pesce, B., Cordon-Cardo, C., and Pandolfi, P.P. 1998. Pten is essential for embryonic development and tumour suppression. Nat. Genet. 19: 348-355.

Groszer, M., Erickson, R., Scripture-Adams, D.D., Lesche, R., Trumpp, A., Zack, J.A., Kornblum, H.I., Liu, X., and Wu, H. 2001. Negative regulation of neural stem/progenitor cell proliferation by the Pten tumor suppressor gene in vivo. Science 294: 2186-2189.

Hennings, H., Shores, R., Mitchell, P., Spangler, E.F., and Yuspa, S.H. 1985. Induction of papillomas with a high probability of conversion to malignancy. Carcinogenesis 6: 1607-1610.

Hildenbrand, C., Burgdorf, W.H., and Lautenschlager, S. 2001. Cowden syndrome-diagnostic skin signs. Dermatology 202: 362-366.

Ikeda, T., Yoshinaga, K., Suzuki, A., Sakurada, A., Ohmori, H., and Horii, A. 2000. Anticorresponding mutations of the KRAS and PTEN genes in human endometrial cancer. Oncol. Rep. 7: 567-570.

Ise, K., Nakamura, K., Nakao, K., Shimizu, S., Harada, H., Ichise, T., Miyoshi, J., Gondo, Y., Ishikawa, T., Aiba, A., et al. 2000. Targeted deletion of the H-ras gene decreases tumor formation in mouse skin carcinogenesis. Oncogene 19: 2951-2956.

Kubo, Y., Urano, Y., Hida, Y., and Arase, S. 1999. Lack of somatic mutation in the PTEN gene in squamous cell carcinomas of human skin. J. Dermatol. Sci. 19: 199-201.

Li, J., Yen, C., Liaw, D., Podsypanina, K., Bose, S., Wang, S.I., Puc, J., Miliaresis, C., Rodgers, L., McCombie, R., et al. 1997. PTEN, a putative protein tyrosine phosphatase gene mutated in human brain, breast, and prostate cancer. Science 275: 1943-1947.

Liaw, D., Marsh, D.J., Li, J., Dahia, P.L., Wang, S.I., Zheng, Z., Bose, S., Call, K.M., Tsou, H.C., Peacocke, M., et al. 1997. Germline mutations of the PTEN gene in Cowden disease, an inherited breast and thyroid cancer syndrome. Nat. Genet. 16: 64-67.

Marsh, D.J., Dahia, P.L., Zheng, Z., Liaw, D., Parsons, R., Gorlin, R.J., and Eng, C. 1997. Germline mutations in PTEN are present in BannayanZonana syndrome. Nat. Genet. 16: 333-334.

Nagase, H., Mao, J.H., and Balmain, A. 2003. Allele-specific Hras mutations and genetic alterations at tumor susceptibility loci in skin carcinomas from interspecific hybrid mice. Cancer Res. 63: 4849-4853.

Perez-Losada, J. and Balmain, A. 2003. Stem-cell hierarchy in skin cancer. Nat. Rev. Cancer 3: 434-443.

Podsypanina, K., Ellenson, L.H., Nemes, A., Gu, J., Tamura, M., Yamada, K.M., Cordon-Cardo, C., Catoretti, G., Fisher, P.E., and Parsons, R. 1999. Mutation of Pten/Mmacl in mice causes neoplasia in multiple organ systems. Proc. Nat1. Acad. Sci. 96: 1563-1568.

Quintanilla, M., Brown, K., Ramsden, M., and Balmain, A. 1986. Carcinogen-specific mutation and amplification of Ha-ras during mouse skin carcinogenesis. Nature 322: 78-80.

Segrelles, C., Ruiz, S., Perez, P., Murga, C., Santos, C., Budunova, I.V., Martinez, J., Larcher, F., Slaga, T.J., Gutkind, J.S., et al. 2002. Functional roles of Akt signaling in mouse skin tumorigenesis. Oncogene 21: 53-64.

Serrano, M., Lin, A.W., McCurrach, M.E., Beach, D., and Lowe, S.W. 1997. Oncogenic ras provokes premature cell senescence associated with accumulation of p53 and p16 INK4a. Cell 88: 593-602.

Shan, X., Czar, M.J., Bunnell, S.C., Liu, P., Liu, Y., Schwartzberg, P.L., and Wange, R.L. 2000. Deficiency of PTEN in Jurkat T cells causes constitutive localization of Itk to the plasma membrane and hyperresponsiveness to CD3 stimulation. Mol. Cell. Biol. 20: 69456957.

Stambolic, V., Suzuki, A., de la Pompa, J.L., Brothers, G.M., Mirtsos, C., Sasaki, T., Ruland, J., Penninger, J.M., Siderovski, D.P., and Mak, T.W. 1998. Negative regulation of PKB/Akt-dependent cell survival by the tumor suppressor PTEN. Cell 95: 29-39.

Steck, P.A., Pershouse, M.A., Jasser, S.A., Yung, W.K., Lin, H., Ligon, A.H., Langford, L.A., Baumgard, M.L., Hattier, T., Davis, T., et al. 
1997. Identification of a candidate tumour suppressor gene, MMAC1, at chromosome 10q23.3 that is mutated in multiple advanced cancers. Nat. Genet. 15: 356-362.

Suzuki, A., Yamaguchi, M.T., Ohteki, T., Sasaki, T., Kaisho, T., Kimura, Y., Yoshida, R., Wakeham, A., Higuchi, T., Fukumoto, M., et al. 2001. $\mathrm{T}$ cell-specific loss of Pten leads to defects in central and peripheral tolerance. Immunity 14: 523-534.

Suzuki, A., Itami, S., Ohishi, M., Hamada, K., Inoue, T., Komazawa, N., Senoo, H., Sasaki, T., Takeda, J., Manabe, M., et al. 2003. Keratinocyte-specific Pten deficiency results in epidermal hyperplasia, accelerated hair follicle morphogenesis and tumor formation. Cancer Res. 63: 674-681.

Tsao, H., Zhang, X., Fowlkes, K., and Haluska, F.G. 2000. Relative reciprocity of NRAS and PTEN/MMAC1 alterations in cutaneous melanoma cell lines. Cancer Res. 60: 1800-1804.

Tsao, H., Goel, V., Wu, H., Yang, G., and Haluska, F.G. 2004. Genetic interaction between NRAS and BRAF mutations and PTEN/MMAC1 inactivation in melanoma. J. Invest. Dermatol. 122: 337-341.

Vivanco, I. and Sawyers, C.L. 2002. The phosphatidylinositol 3-Kinase AKT pathway in human cancer. Nat. Rev. Cancer 2: 489-501. 


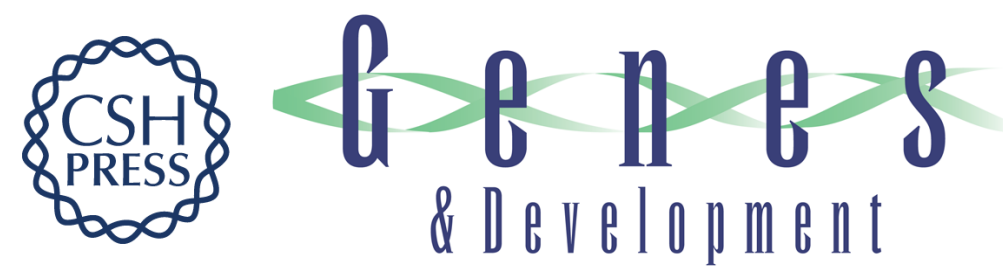

\section{Mutually exclusive mutations of the Pten and ras pathways in skin tumor progression}

Jian-Hua Mao, Minh D. To, Jesus Perez-Losada, et al.

Genes Dev. 2004, 18:

Access the most recent version at doi:10.1101/gad.1213804

References

This article cites 31 articles, 8 of which can be accessed free at:

http://genesdev.cshlp.org/content/18/15/1800.full.html\#ref-list-1

\section{License}

Email Alerting

Receive free email alerts when new articles cite this article - sign up in the box at the top Service right corner of the article or click here.

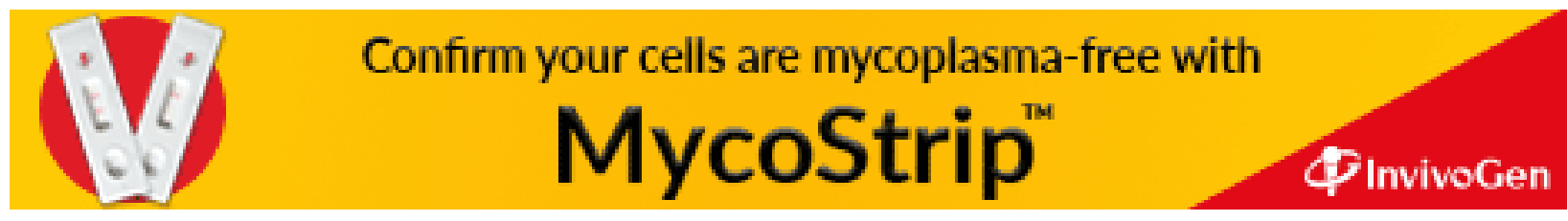

